\title{
BMJ Open Protocol for the development and acceptability of a fertility-related decision aid for young women with breast cancer in Portugal
}

Vânia Gonçalves, ${ }^{1}$ Luzia Travado, ${ }^{2}$ Pedro Lopes Ferreira, ${ }^{3}$ Gwendolyn Quinn ${ }^{4}$

To cite: Gonçalves V, Travado L, Ferreira PL, et al. Protocol for the development and acceptability of a fertilityrelated decision aid for young women with breast cancer in Portugal. BMJ Open 2019;9:e030690. doi:10.1136/ bmjopen-2019-030690

- Prepublication history for this paper is available online. To view these files, please visit the journal online (http://dx.doi. org/10.1136/bmjopen-2019030690).

Received 26 March 2019 Revised 17 June 2019 Accepted 25 June 2019

A Check for updates

(c) Author(s) (or their employer(s)) 2019. Re-use permitted under CC BY-NC. No commercial re-use. See rights and permissions. Published by BMJ.

${ }^{1}$ Centre for Health Studies and Research of the University of Coimbra (CEISUC), Coimbra,

Portugal

${ }^{2}$ Psycho-oncology, Neuropsychiatry Unit, Champalimaud Foundation, Lisbon, Portugal

${ }^{3}$ Centre for Health Studies and Research of the University of Coimbra (CEISUC), Faculty of Economics, University of Coimbra, Coimbra, Portugal ${ }^{4}$ Population Health, NYU Langone Health, New York City, New York, USA

Correspondence to Dr Vânia Gonçalves; vmo.goncalves@hotmail.com

\section{ABSTRACT}

Introduction Young patients with breast cancer may face impaired fertility due to cancer treatments, which often leads to complex fertility decisions. To aid fertility decision-making, it is crucial that women have access to high-quality information; however, their fertility information needs are often unmet. Decision aids (DAs) are educational materials to assist with decision-making, by addressing individual values and preferences. In oncofertility, DAs may constitute a valuable resource to help patients obtain information and make better informed decisions. This paper reports on the protocol of the development and transcreation of a fertility-related DA booklet to support young Portuguese patients with breast cancer, originally developed and validated for an Australian audience. Methods and analysis Recent literature on clinical guidelines will be reviewed. A summary of these guidelines will be created and will inform the first round of DAs revisions. A forward translation process will translate the DA from Australian English to Portuguese. A multidisciplinary Portuguese experts panel will revise and give feedback on the scientific and cultural aspects of the DA content for Portuguese audience. Next, a backward translation process will assess content equivalence between the original and the final adapted version. Finally, Learner Verification (LV) will be used in a qualitative study of young patients with breast cancer and their partners. Two focus groups with 6-10 participants each will be conducted with: (1) recently diagnosed young patients with breast cancer; (2) breast cancer survivors and (3) their partners. Results from the DA acceptability assessment will inform its final version. Data will be analysed using content analysis and constant comparison method to identify key themes/textual units related to LV.

Ethics and dissemination Ethical approval was granted by the Portuguese Institute of Oncology Porto. Results will be disseminated through peer-reviewed journals and presented at scientific meetings for academic and health professionals audiences.

\section{INTRODUCTION}

Oncofertility is currently a frontline research topic and a vital issue in clinical care for young patients with breast cancer. As cancer survivors live longer, it is important to consider long-term effects of cancer treatment on

\section{Strengths and limitations of this study}

To our knowledge, this is the first study in Portugal to develop and validate an evidence-based decision aid (DA) specifically designed for young patients with breast cancer to support and facilitate the complex fertility decision-making process that women face during their cancer trajectory.

- Results of this study will provide evidence of its acceptability to women at different stages of the cancer trajectory and their significant others/partners who may play a crucial role during the process of fertility decision-making.

- Results of this study will inform an emerging field of research in Portugal and highlight the importance of fertility for young patients with breast cancer, by facilitating the implementation of fertility discussions in clinical settings.

- This study will be limited to Portuguese-speaking women; however, since there are few validated DAs for young patients with breast cancer worldwide, results of its acceptability and validation will inform a growing, but still scarce, field of research.

- The use of qualitative methods in the focus groups and learner verification reduces generalisability. The exclusive focus on patients with breast cancer also limits application to other cancer sites.

fertility and offer optimal ways to improve quality of life (QoL). ${ }^{1}$ Often when young patients with breast cancer are diagnosed, they have not initiated or completed their childbearing goals. $^{2}$ Concomitantly, cancer treatment may impair fertility, permanently or temporarily. ${ }^{3}$ In this context, consideration of patients' fertility needs and the possibility to retain genetic reproductive potential should be included in the management of breast cancer as advocated by diverse professional bodies and clinical practice guidelines. ${ }^{45}$ Such guidelines offer evidence for healthcare professionals and patients decision-making regarding appropriate, safe and cost-effective fertility care. Further, guidelines 
highlight healthcare professionals' duty to provide timely information on cancer treatments likely threat to future fertility and to discuss fertility preservation options with women of childbearing age. Those options offer strategies to preserve women's fertility and the ability to have genetic children. ${ }^{3}$

Strong evidence suggests that fertility and other reproductive health issues are important for women of reproductive age and a significant QoL concern, ${ }^{26-8}$ adding to the emotional burden of the cancer diagnosis for these young women. ${ }^{9}$ Previous work on reproductive attitudes of young breast cancer survivors showed novel data on Portuguese women, corroborating the evidence from other countries that fertility is an important issue for these survivors, namely for those who are young and childless before the diagnosis. ${ }^{8}$ Compared with a sample of women from the USA, no significant differences were found among Portuguese women on the importance of fertility issues after a breast cancer diagnosis. These results are corroborated by other studies, ${ }^{10} 11$ demonstrating that women value these issues regardless of culture, background or the health system they belong to. ${ }^{8}$ For some women, fertility concerns are important enough to influence or even change therapeutic decisions. ${ }^{8}$

Currently, there are several available fertility preservation techniques to assist young patients with breast cancer in preserving their fertility. These options include embryo and oocyte freezing and the experimental option of ovarian tissue cryopreservation. National guidelines typically suggest that these methods be used prior to the initiation of gonadotoxic treatment, such as chemotherapy, although some providers and patients attempt preservation methods during a break in or immediately following treatment. ${ }^{12}$ Adoption and third party reproduction are other options for women who were not able to use fertility preservation techniques prior to the start of cancer treatment or for whom techniques were unsuccessful. However, all options, even simply waiting to see if fertility potential becomes impaired, typically entails a series of complex decisions for patients and healthcare professionals. ${ }^{13}{ }^{14}$ These decisions are intricate for a variety of reasons including the uncertainty about one's health and lifespan, the interest in raising a child with a partner, the disposition of stored gametes, one's religious and moral values associated with the use of assisted reproductive technologies, financial issues (however, in Portugal, the National Health System covers fertility preservation procedures for patients with cancer, in public health institutions ${ }^{15}$ ), medical issues and the need to make high-quality informed decisions. ${ }^{2} 131617$ The demand to consider a fertility decision usually occurs within a chaotic context for the patient: the emotional turmoil of receiving a cancer diagnosis and the vital need of starting a cancer treatment. Furthermore, guidelines suggest that established and experimental fertility preservation options be used prior to commencement of gonadotoxic cancer treatment. ${ }^{18}$ Despite the complexity of decision-making, it is imperative that women have access to high-quality information to aid decision-making. Several studies have shown that young patients with cancer consider fertility information a priority; however, their fertility-related information needs are often unmet. ${ }^{6719}$ Particularly in Portugal, findings also reported that some survivors did not recall any discussions about fertility with their health providers or were not satisfied with the explanations given about potential infertility. ${ }^{8}$ However, young women with breast cancer wish to receive written materials to inform their choices about fertility preservation. ${ }^{20}$ Moreover, women desire to thoroughly understand the potential of cancer treatment effects on fertility, available preservation options and potential risks associated with these options. ${ }^{21}$ Research suggests that women benefit from and have reduced remorse and regret when provided opportunities to learn about potential infertility, even if no options are available or elected. ${ }^{22}$ Decisional conflict, which is the state of uncertainty about the course of action to be taken, tends to be associated with emotional distress, future regret or/and blame and delayed decision-making. ${ }^{14}$

Decision Aids (DAs) are educational materials designed to assist with treatment decision-making, by addressing individual values and preferences, ${ }^{6}$ and are particularly helpful in situations when there is limited time to make the decision. ${ }^{23}$ DAs help make the decision explicit, describe options available and assist patients understanding of options as well as their possible benefits and harms. DAs assist patients in considering the options from a personal perspective, allowing them to participate with their health provider in shared decision-making. ${ }^{24}$ Particularly for an oncofertility context, DAs may constitute a valuable resource to help patients obtain more fertility-related information such as documented by a recent systematic review, which concluded that fertility-related DAs for patients with cancer can be effective complements to current fertility care by increasing fertility information satisfaction and help patents to make better-informed decisions. ${ }^{25}$ However, validated tools to support patients in this process, including DAs, are scarce.$^{26}$ One of the first fertility-related DAs for young patients with breast cancer was developed and validated by Peate $e t$ at in Australia. Compared with standard care, the fertility-related DA was shown to reduce decisional conflict about fertility options and reduce decisional regret about fertility treatments. In addition, women who received the DA reported more satisfaction with fertility information and rated it more helpful than women who received standard information. ${ }^{6}$ The DA also improved fertility-related knowledge. Other evaluated DAs, include one for young women with breast cancer in Dutch ${ }^{27}$ and another DA in German for young female patients with cancer. ${ }^{28}$ Two DAs for young female patients with cancer in English are currently under evaluation. ${ }^{29}{ }^{30}$ Although, oncofertility in Portugal is still in the developmental stage, ${ }^{31}$ findings from a previous study suggest that it is imperative to include fertility counselling in breast cancer management and initiate efforts to address the unmet fertility information needs 
of Portuguese patients with cancer. ${ }^{8}$ Based on the Peate fertility-related DA, ${ }^{6}$ updated in $2016,{ }^{32}$ we aim to transcreate and validate the fertility-related DA for young Portuguese patients with breast cancer. Merely translating cancer educational materials, in this particular case from Australian English to Portuguese, has been shown to be insufficient to improve patients' knowledge. ${ }^{33}$ Therefore, our study aims to transcreate the original DA to a Portuguese audience, which is a process that goes beyond direct translation of the text, and includes culturally relevant themes, images and context, ensuring that the text is reconstructed with cultural relevance to meet the young Portuguese breast cancer patients' informational needs. ${ }^{34}$ It includes not only the translation but also a cultural adaptation. The decision to use a developed and validated fertility-related DA specifically designed for young patients with breast cancer to guide our work was based in its efficacy, previously documented in this population. Furthermore, one of the advantages of this tool is that its original language is English, a language in which the majority of the research team is fluent. To our knowledge, our study is a pioneer in Portugal, since there are no validated fertility-related DAs specifically designed for young patients with breast cancer in Portuguese.

Our project entails the development, transcreation and validation of a DA to support young Portuguese patients with breast cancer who are at the potential stage of making fertility preservation decisions before the initiation of their cancer treatment. The project consists of a longitudinal prospective study, to be conducted in a two-phase process: phase 1) development and transcreation of a fertility-related DA booklet to support young Portuguese women diagnosed with breast cancer, originally developed, validated and updated for an Australian audience, ${ }^{632}$ phase 2 ) validation of the fertility-related DA for a Portuguese audience and to establish efficacy of the tool. The present paper reports on the protocol of the project's phase 1 , the fertility-related DA development and transcreation to a Portuguese audience.

\section{METHODS AND ANALYSIS}

\section{DA content development to a Portuguese audience}

The development of the Peate DA used a framework guided by the International Patient Decision Aid Standards collaboration, ${ }^{35}$ and was validated in a sample of women aged between 18 and 40 years (inclusive). ${ }^{21}$ The DA is a booklet that includes background information about breast cancer; its treatments and impact on fertility; a summary of female fertility and a description of current fertility preservation options, including relevant scientific information, financial costs and associated risks with each fertility preservation method. In addition to its informational content, the DA also has an interactive portion, consisting of a set of value clarification exercises, which present the advantages and disadvantages of each treatment option. Women are asked to rate each option in light of their values and life situation, using a 5-point scale ranging from 'I am leaning towards (fertility option) to 'I am not leaning towards (fertility option)'. Collectively, the DA provides the patient with a personalised summary of each fertility option, enabling and facilitating decision-making about fertility preservation. ${ }^{6} 32$

The transcreation process will be informed by three phases: (1) our previous work in the field of fertility preservation $^{2818} ;(2)$ the completion of a narrative review of current guidelines for fertility preservation; (3) a narrative review of DAs for young women with cancer. The narrative reviews will follow standard Preferred Reporting Items for Systematic Reviews and Meta-Analyses methods using Boolean search operators and will include quantitate, qualitative and mixed methods peer-reviewed publications by searching the databases: Medline, EMBASE, ISI Web of Knowledge, PubMed and PsychLit. ${ }^{36}$ A summary of our previous findings and the narrative reviews will be created and guide the first round of revisions to the DA. Next, a process of forward translation, in which the content of the original fertility-related DA for early breast cancer patients will be translated to European Portuguese language by two independent professional translators, will be employed. Finally, a multidisciplinary panel of Portuguese experts in the field (oncologists, fertility specialists, gynaecologists, psycho-oncologists and nurses), will revise the scientific and cultural aspects of the DA content for a Portuguese context. The summary from the experts' feedback will be analysed by the research team and any incongruences will be clarified with the experts, if necessary. The research team will discuss all feedback until consensus is reached. Then, we will employ a process of back translation, in which the final version will be back translated from Portuguese to Australian-English to assess content equivalence between the original and the final adapted version. The adequacy of the DA's content will be assessed for a Portuguese audience, accounting for possible country differences in treatment options, medical management and health system between Australia and Portugal. Lastly, the results from the DA acceptability assessment will inform and lead to the final version of the DA, which will be validated using a randomised controlled trial (RCT) in phase 2. This project began in January 2017 and it will end in December 2021. Figure 1 illustrates the process of study phase 1 .

\section{DA acceptability assessment}

To assess acceptability of the DA, we will use a process called Learner Verification (LV) that assesses elements such as attractiveness, comprehension, cultural acceptability, self-efficacy and persuasion. ${ }^{37} \mathrm{LV}$ is also a quality control technique that helps to ensure the educational materials are suitable for the intended audience and meet patients' learning needs. ${ }^{37} \mathrm{LV}$ is rooted in information processing theory, focusing on the persuasiveness of a health message, providing a systematic process for assessing the intended message of a DA. ${ }^{38}$ In our study, specific components of $\mathrm{LV}$ will be assessed with young Portuguese who were diagnosed with breast cancer, 


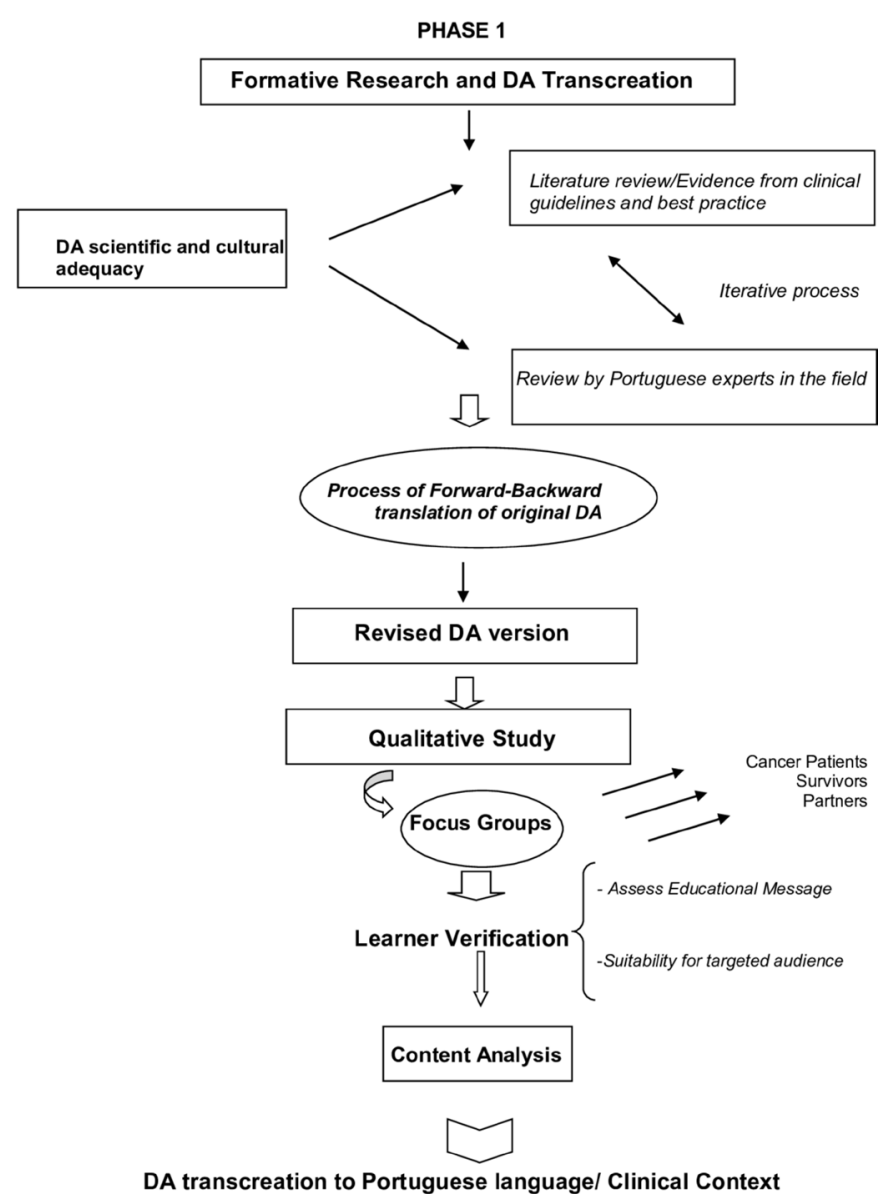

Figure 1 Study phase 1 process. DA, decision aid.

including current patients, survivors and partners. The LV components include message attraction (such as 'Does the DA appeal to young Portuguese breast cancer survivors/patients/partners?'), comprehension (such as 'Do young Portuguese breast cancer survivors/patients/ partners understand the information in the DA?'), self-efficacy (such as 'Are young Portuguese breast cancer survivors/patients/partners capable of making a decision?'), cultural acceptability (such as 'Do young Portuguese breast cancer survivors/patients/partners perceive the message to be relevant and acceptable?') and persuasion (such as 'Does the DA convince young Portuguese breast cancer survivors/patients/partners that need for a decision is relevant ?'). ${ }^{38}$

\section{Design and sample}

A qualitative study will be used. The acceptability of the DA will be assessed with focus groups from a breast unit in a national oncology hospital in Portugal. We will enrol three populations of the intended audience: (1) recently diagnosed patients with breast cancer aged 18-45 at diagnosis and between 2-6 months since the initial diagnosis; (2) breast cancer survivors diagnosed at age 18-45 and not receiving active cancer treatment other than aromatase inhibitors and (3) partners (of any age) of patients or survivors aged 18-45 years at the time of diagnosis. Furthermore, instead of a romantic partner, the patient may indicate a significant other who is caring for her or cared for her during treatment. The decision to have a group of patients' partners during the development of the DA was based on findings from a previous study that indicated partners often play an important role in shaping women's fertility attitudes and decisions. ${ }^{8}$

For each population of the intended audience, two focus groups will be conducted with 6-10 participants each, since for LV only small samples of the intended audience are needed (6-10 participants in a group).$^{38}$ The goal of the qualitative $\mathrm{LV}$ endeavour is to achieve saturation and thus if saturation is not reached (no new information), we will recruit additional participants as needed. Inclusion criteria for all focus groups will be the requirement to read and write in the Portuguese language and willing to provide written consent.

\section{Analysis}

Data will be analysed using content analysis ${ }^{39}$ and the constant comparison method to identify key themes/ textual units related to LV. We will aim for thematic saturation (where data collection does not generate substantially new information and the range of perspectives appear to be completely covered) between the population specific focus groups. ${ }^{40}$ All focus groups will be audiotaped and verbatim transcripts coded for key themes with the goal of at least $85 \%$ intercoder reliability between at least two coders.

\section{Patient and public involvement}

Neither patients nor public were involved in the development of this study design but they will be involved in the development and validation of the DA.

\section{Dissemination}

Results will be disseminated through peer-reviewed journals and presented at relevant scientific meetings for academic and health professionals audiences.

\section{DISCUSSION}

Oncofertility is widely accepted and supported by the scientific community as a key component of young women's oncological care and QoL. However, in clinical settings, its implementation is still suboptimal. A recent study conducted in Portugal and USA showed similar priorities and concerns regarding fertility and childbearing for young patients with breast cancer. Lack of fertility discussions and dissatisfaction with fertility information provided by healthcare professionals were reported. ${ }^{8}$ DAs are educational tools that may have the potential to facilitate the delivery of fertility information and aid in the complex decisional pathway that the patient and health professionals have to go through. A recent systematic review of decision tools for fertility supports the view that the use of these tools in clinical settings should be encouraged, since DAs were found to be highly useful, leading to discussions about fertility 
preservation and less decisional regret. ${ }^{2541}$ The number of available written materials about fertility preservation for young patients with cancer is growing ${ }^{27} 28$ as online fertility-related information from different organisations. ${ }^{42}$ However, there are few validated DAs for young patients with breast cancer worldwide; to our knowledge, they are non-existent in Portugal.

Our project represents the first study in Portugal to develop and validate a DA for young patients with breast cancer who speak Portuguese to support and facilitate the fertility decision-making process during a very difficult and stressful period of women's cancer trajectory, the diagnostic phase. Providing the clinical cancer care team with an evidence-based tool to administer to all women during this period will encourage healthcare providers to have more fertility discussions with their patients, helping them to make appropriate and timely referrals for fertility specialists, and ultimately, raising awareness for the inclusion of fertility and childbearing within their clinical practice.

Future research integrated into this project includes a RCT developed to validate and assess DAs efficacy in a sample of young women with breast cancer (phase 2). Based on findings using its original version in Australia ${ }^{6}$ and from the literature in general, ${ }^{25}$ we anticipate that the DA will be an acceptable and useful tool for these group of patients during the fertility decision-making process, such as contributing to an increase in their fertility preservation knowledge and satisfaction with the information, as well as, helping to decrease the emotional burden of cancer. The innovative nature of this study addresses gaps in the scientific and clinical practice cited above, targeting an emerging field of research in Portugal, with the expectation of improving clinical practice, patient-provider communication and knowledge in oncofertility.

Contributors VG conceived the idea, contributed to the design of the study and protocol, coordinates the decision aid development, secured funding and drafted this manuscript. LT participates in the decision aid development and in the decision aid revision. PLF provides intellectual and scientific insight during the decision aid development and participates in the decision aid revision. GQ contributed to the design of the study, provided intellectual and scientific input during the development of the study protocol and grant application process. In addition, GQ provides intellectual and scientific input during the development of the decision aid. All authors reviewed and approved the final version of the protocol.

Funding This project was funded by Foundation for Science and Technology of Portugal Tecnologia (FCT), under grant agreement no IF/00793/2015.

Competing interests None declared.

Patient consent for publication Not required.

Ethics approval Ethical approval was obtained from the Ethics Committee of the Portuguese Institute of Oncology of Porto (IP0-Porto) (CES IP0: 34/2018).

Provenance and peer review Not commissioned; externally peer reviewed.

Open access This is an open access article distributed in accordance with the Creative Commons Attribution Non Commercial (CC BY-NC 4.0) license, which permits others to distribute, remix, adapt, build upon this work non-commercially, and license their derivative works on different terms, provided the original work is properly cited, appropriate credit is given, any changes made indicated, and the use is non-commercial. See: http://creativecommons.org/licenses/by-nc/4.0/.

\section{REFERENCES}

1. Ruddy KJ, Gelber S, Ginsburg ES, et al. Menopausal symptoms and fertility concerns in premenopausal breast cancer survivors: a comparison to age- and gravidity-matched controls. Menopause 2011;18:105-8.

2. Gonçalves V, Sehovic I, Quinn G. Childbearing attitudes and decisions of young breast cancer survivors: a systematic review. Hum Reprod Update 2014;20:279-92.

3. Tschudin S, Bitzer J. Psychological aspects of fertility preservation in men and women affected by cancer and other life-threatening diseases. Hum Reprod Update 2009;15:587-97.

4. Oktay K, Harvey BE, Partridge AH, et al. Fertility preservation in patients with cancer: ASCO Clinical practice guideline update. $J$ Clin Oncol 2018;36:1994-2001.

5. Almeida Santos AT, Sousa G, Teixeira A, et al. Recomendações para a preservação do potencial reprodutivo no doente oncológico. $R P O$ 2016;2:5-24.

6. Peate M, Meiser B, Friedlander M, et al. It's now or never: fertilityrelated knowledge, decision-making preferences, and treatment intentions in young women with breast cancer--an Australian fertility decision aid collaborative group study. J Clin Oncol 2011;29:1670-7.

7. Benedict $C$, Shuk E, Ford JS. Fertility issues in adolescent and young adult cancer survivors. J Adolesc Young Adult Oncol 2016;5:48-57.

8. Gonçalves V, Hudson J, Canavarro MC, et al. Childbearing across borders: fertility and parenthood attitudes and decisions among breast cancer survivors in USA and Portugal. Breast 2018;40:16-22.

9. Travado L, Rowland J. Supportive care and Psycho-oncology Issues During and Beyond Diagnosis and Treatment. In: Pagani O, Gentilini $\mathrm{O}$, Partridge $\mathrm{A}$, eds. Breast cancer in young women: Springer.

10. Urech C, Ehrbar V, Boivin J, et al. Knowledge about and attitude towards fertility preservation in young female cancer patients: a cross-sectional online survey. Hum Fertil 2018;21:45-51.

11. Ehrbar V, Urech C, Alder J, et al. Decision-making about fertility preservation-qualitative data on young cancer patients' attitudes and needs. Arch Womens Ment Health 2016;19:695-9.

12. Lambertini M, Del Mastro L, Pescio MC, et al. Cancer and fertility preservation: international recommendations from an expert meeting BMC Med 2016;14:1.

13. Quinn GP, Vadaparampil ST, Lee JH, et al. Physician referral for fertility preservation in oncology patients: a national study of practice behaviors. J Clin Oncol 2009;27:5952-7.

14. Kim J, Deal AM, Balthazar U, et al. Fertility preservation consultation for women with cancer: are we helping patients make high-quality decisions? Reprod Biomed Online 2013;27:96-103.

15. Assembleia da República Portuguesa. Resolução $n^{\circ} 112 / 2015$, de 7 de Agosto. Diário da República $1^{\text {a }}$ série. 2015;153:5505.

16. Quinn GP, Vadaparampil ST. More research, more responsibility: the expansion of duty to warn in cancer patients considering fertility preservation. Am J Obstet Gynecol 2013;209:98-102.

17. Jones G, Hughes J, Mahmoodi N, et al. What factors hinder the decision-making process for women with cancer and contemplating fertility preservation treatment? Hum Reprod Update 2017;23:433-57.

18. Gonçalves V, Quinn GP. Review of fertility preservation issues for young women with breast cancer. Hum Fertil 2016;19:152-65.

19. Bailey AP, Guinsburg ES. Fertility preservation options for females. In: Quinn G, Vadaparampil S, eds. Reproductive health and cancer in adolescents and young adults. Advances in experimental medicine and biology. Netherlands: Springer, 2012:9-28.

20. Hill KA, Nadler T, Mandel R, et al. Experience of young women diagnosed with breast cancer who undergo fertility preservation consultation. Clin Breast Cancer 2012;12:127-32.

21. Peate M, Meiser B, Cheah BC, et al. Making hard choices easier: a prospective, multicentre study to assess the efficacy of a fertilityrelated decision aid in young women with early-stage breast cancer. Br J Cancer 2012;106:1053-61.

22. Letourneau JM, Ebbel EE, Katz PP, et al. Pretreatment fertility counseling and fertility preservation improve quality of life in reproductive age women with cancer. Cancer 2012;118:1710-7.

23. Nicholas Z, Butow P, Tesson S, et al. A systematic review of decision aids for patients making a decision about treatment for early breast cancer. Breast 2016;26:31-45.

24. Stacey D, Légaré F, Col NF, et al. Decision aids for people facing health treatment or screening decisions. Cochrane Database Syst Rev 2014:CD001431.

25. Anazodo A, Laws P, Logan S, et al. How can we improve oncofertility care for patients? A systematic scoping review of current international practice and models of care. Hum Reprod Update 2019;25:159-79.

26. Quinn G, Vadaparampil S, Sehovic I, et al. Patient and Family tools to aid in education and decision-making about oncofertility. In: 
Woodruff TK, ed. Oncofertility communication: sharing information and building relationships across disciplines. New York: Springer Science+Business Media, 2014:35-47.

27. Garvelink MM, Ter Kuile MM, Louwé LA, et al. Feasibility and effects of a decision aid about fertility preservation. Hum Fertil 2017;20:104-12.

28. Ehrbar V, Urech C, Rochlitz C, et al. Fertility preservation in young female cancer patients: development and pilot testing of an online decision aid. J Adolesc Young Adult Oncol 2018;7:30-6.

29. Jones GL, Hughes J, Mahmoodi N, et al. Observational study of the development and evaluation of a fertility preservation patient decision aid for teenage and adult women diagnosed with cancer: the cancer, fertility and me research protocol. BMJ Open 2017;7:e013219.

30. Woodard TL, Hoffman AS, Crocker LC, et al. Pathways: patientcentred decision counselling for women at risk of cancer-related infertility: a protocol for a comparative effectiveness cluster randomised trial. BMJ Open 2018;8:e019994.

31. Silva C, Almeida-Santos AT, Melo C, et al. Decision on fertility preservation in cancer patients: development of information materials for healthcare professionals. J Adolesc Young Adult Oncol 2017;6:353-7.

32. Peate M, Butow P. Fertility-related choices: a decision aid for younger women with early breast cancer. Camberwell: Breast Cancer Network Australia, 2016

33. Solomon FM, Eberl-Lefko AC, Michaels M, et al. Development of a linguistically and culturally appropriate booklet for Latino cancer survivors: lessons learned. Health Promot Pract 2005;6:405-13.
34. Rivera YM, Vélez $\mathrm{H}$, Canales $\mathrm{J}$, et al. When a common language is not enough: transcreating cancer 101 for communities in Puerto Rico. Journal of Cancer Education 2016;31:776-83.

35. Elwyn G, O'Connor A, Stacey D, et al. Developing a quality criteria framework for patient decision aids: online international Delphi consensus process. BMJ 2006;333:417.

36. Siddaway AP, Wood AM, Hedges LV. How to do a systematic review: a best practice guide for conducting and reporting narrative reviews, meta-analyses, and meta-syntheses. Annu Rev Psychol 2019;70:747-70.

37. Doak LG, Doak CC, Meade CD. Strategies to improve cancer education materials. Oncol Nurs Forum 1996;23:1305-12.

38. Vadaparampil ST, Quinn GP, Gjyshi A, et al. Development of a brochure for increasing awareness of inherited breast cancer in black women. Genet Test Mol Biomarkers 2011;15:59-67.

39. Hsieh HF, Shannon SE. Three approaches to qualitative content analysis. Qual Health Res 2005;15:1277-88.

40. Morgan D. Focus groups in qualitative research. 2 nd edn. Thousand Oaks: Sage, 1997.

41. Ehrbar V, Urech C, Tschudin S. Fertility decision-making in cancer patients - current status and future directions. Expert Rev Qual Life Cancer Care 2018;3:113-9.

42. de Man AM, Rashedi A, Nelen W, et al. Female fertility in the cancer setting: availability and quality of online health information. Hum Fertil 2018:1-9. 